\title{
Baffling telemetry detections can be useful: an acoustic receiver design to monitor organisms along reserve boundaries and ecotones
}

\author{
Matthew S. Kendall ${ }^{*}$, Mark E. Monaco ${ }^{1}$ and Arliss Winship ${ }^{1,2}$
}

\begin{abstract}
Background: Many biotelemetry studies seek to detect movement of organisms across reserve boundaries or between adjacent habitat areas. Our objective was to enhance this capability in studies of aquatic organisms that are tagged with acoustic transmitters and tracked by passive data loggers. We installed an experimental shroud on a commercially available telemetry receiver. The shroud was designed to baffle incoming signals from transmitters along one hemisphere of the receiver and therefore more conclusively determine which side of a boundary line that a tagged organism occupies.

Results: Range testing of shroud effectiveness was conducted along the border of a marine-protected area in a coral reef ecosystem. A transmitter of the type implanted into reef fish was deployed in various directions, distances, and landscape settings relative to 11 shrouded receivers. There was a significant difference in the detectability of transmissions on the shrouded versus unshrouded side of the receivers. On the unshrouded hemisphere of the receiver, 75-100\% of transmissions within $100 \mathrm{~m}$ were detected and maximum effective detection range was $\sim 180 \mathrm{~m}$. On the shrouded hemisphere of the receiver, detections were rare at any distance with a maximum of $12 \%$ of transmissions recorded even as close as $60 \mathrm{~m}$ away.

Conclusions: The shroud modified the detection area of a standard omni-directional receiver into a hemi-directional receiver better able to detect transboundary presence. The approach is useful for applications that require detection of simple boundary-crossing events using a minimal number of receivers.
\end{abstract}

Keywords: Array, Boundary crossing, Fish movement, Marine-protected area, Tracking

\section{Background}

A key question in marine-protected area (MPA) design and performance is estimating the frequency and duration of fish movement across borders [1]. The tendency of fishermen to "fish the line" just outside MPA boundaries necessitates a detailed understanding of the spatial movements of fish relative to MPA borders [2]. There are a range of acoustic telemetry approaches now being utilized to address this question, each with their particular

\footnotetext{
${ }^{*}$ Correspondence: matt.kendall@noaa.gov

${ }^{1} \mathrm{NOAA} / \mathrm{NOS} / \mathrm{NCCOS} / \mathrm{CCMA}$ /Biogeography Branch, 1305 East West

Highway, Silver Spring, MD 20910, USA

Full list of author information is available at the end of the article
}

suite of strengths and limitations. Active tracking of fish implanted with acoustic transmitters using a directional hydrophone offers precise positioning and avoids the need for a costly array of many acoustic receivers, but of course requires presence of researchers and boats to acquire each position (e.g., [3]). Arrays of multiple acoustic receivers and data loggers deployed in grid or gate formations with non-overlapping detection ranges offer relatively automated monitoring but yield only approximate positions based on receiver range [4]. Moderate densities of three or more acoustic receivers deployed with overlapping detection ranges can be used to estimate approximate position or activity centers based on the detection rate of relatively 
stationary transmitters heard at multiple receivers using linear [5] and sigmoid [6] relationships between detection rate and transmission distance. High densities of timesynchronized receivers have also been used along with time of arrival data from transmitters to calculate position within 1-5 m [7-9]. However, all the techniques capable of discriminating fine-scale boundary crossing require close spacing of three or more receivers to ensure overlap in the detection range, and in these systems, transmitter position can only be calculated in the area of overlap. This number of receivers increases hardware costs, deployment, maintenance and recovery logistics, as well as computational requirements. The estimated positions provide a wealth of habitat utilization information that may be extraneous to simply detect positions relative to linear boundaries associated with MPAs.

We sought to identify a technique that would sensitively identify presence/absence along one side of a boundary but eliminate the need for extensive arrays of closely spaced hydrophones and complex data processing required by approaches presently available. Experiments were conducted to convert standard omni-directional acoustic receivers into hemi-directional receivers that could only detect fish presence in a specified hemisphere on one side of a receiver. We installed an experimental acoustic shroud over the hydrophone of a commercially available acoustic data logger to test whether it would enable more detailed quantification of boundary-crossing events of tagged fish.

\section{Methods}

\section{Study area}

The experimental system was tested in a natural ecosystem rather than in a highly controlled environment in order to evaluate its real-world potential. The St. John, US Virgin Islands (USVI) study area consists of many small, mangrove-lined bays with scattered patch reefs. At this latitude in the Caribbean $(18.3 \mathrm{~N}, 64.7 \mathrm{~W})$ the trade winds blow consistently from east to west. The Virgin Islands coral reef national Monument (VICRNM) is a no-take MPA that bisects portions of Coral Bay and Round Bay and was the focus of this evaluation [10]. The water column is well mixed. Wave energy and tidal or other currents are always minimal throughout the area. Biotic sounds experience small peaks around dawn and dusk but are uniform at other times [11]. These uniform water characteristics are not believed to be a major source of spatial or temporal variability on transmitter detections $[4,6,12,13]$.

\section{Hemi-directional receivers}

Eleven acoustic receivers were deployed along the VICRNM boundary. All were between 17 and $24 \mathrm{~m}$ depth except the northwestern receiver which was at $6 \mathrm{~m}$. We used the commercially available VR2W model receiver from VEMCO, Amirix Systems Inc. during these experiments. This battery powered hydrophone and data logger is an automated system that has been deployed in a wide diversity of telemetry studies worldwide. The ability of the standard VR2W omni-directional receivers to detect fish movements across the boundary of the Monument was modified by attaching a directional acoustic shroud onto the hydrophone of the receivers. This modification was inspired by prior studies suggesting that materials including mooring equipment near the hydrophone transducer can block acoustic signals [14, VEMCO pers. comm.]. The shroud was constructed from a $15.25-\mathrm{cm}$ diameter (6 inch), solid PVC foam float of the type commonly used in boating and fishing applications. The airfilled foam of the shroud theoretically provided a barrier to acoustic pings from transmitters. The PVC float was cut lengthwise in half to make two shrouds. Each half was partially hollowed out to fit snugly around the top of a VR2W such that the actual transducer on the top of the VR2W would seat into the existing rope channel of the PVC float (Fig. 1). Shrouds had slots drilled through them

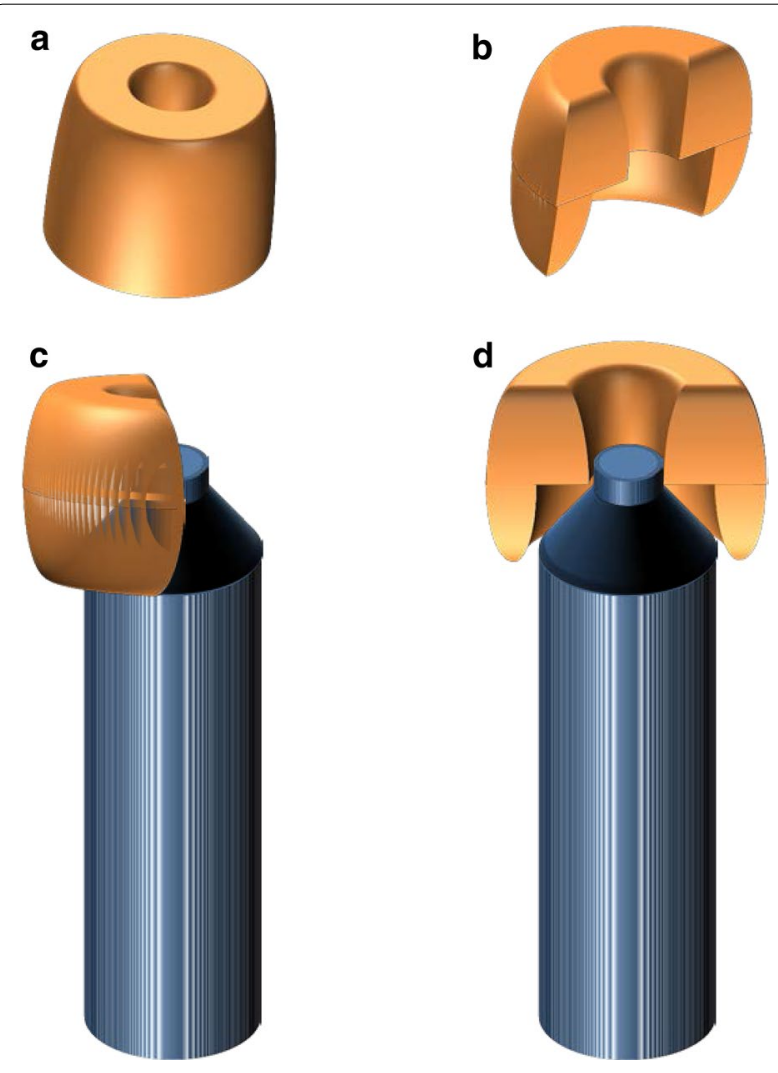

Fig. 1 Design of the acoustic shroud from: a whole PVC float, $\mathbf{b}$ float cut in half and then hollowed out to fit over the top of the VR2W, c side view of shroud mounted on VR2W shown in black, and $\mathbf{d}$ facing view 
to enable tight attachment onto the hydrophone using zip ties through the existing pry bar holes in the VR2W housing. In this arrangement, the shroud theoretically blocks incoming pings from one hemisphere around the receiver but allows normal detection of pings from the open side. As a result an omni-directional receiver is converted into a hemi-directional receiver. The mooring system therefore must maintain the orientation of the shroud along the axis of interest, in this case parallel to the Monument boundary.

The mooring system maintained receivers in a vertical orientation and more importantly, prevented rotation of the shroud off the axis of the Monument boundary to ensure monitoring of signals from the desired direction [14]. This was accomplished by installing two sand screws $\sim 1.5 \mathrm{~m}$ apart along the Monument boundary, and securing them to the sides of the shrouded receiver such that it maintained its directional orientation (Fig. 2). Once installed, divers twisted and pulled on the assembly to confirm that the floats would quickly return the system to its desired orientation. It should be noted that this mooring was effective in our low-wave energy and minimal current environment but a fixed or rigid design may be needed elsewhere. All shrouds were observed to be intact and in place when receivers were retrieved for download at the end of the study. Two unmodified, omnidirectional receivers were deployed at two of the hemidirectional receiver sites as controls but unfortunately both of these experienced component failures, did not record data, and will not be discussed further.

\section{Testing detection range and direction}

Sensitivity of receivers to detect transmitter signals along the VICRNM boundary was determined by deploying a range-test transmitter in a diversity of locations around the modified receivers throughout the study area. In this analysis, a range-test transmitter (for VEMCO V7-4L transmitters) with a $\sim 15 \mathrm{~s}$ transmission interval was deployed $\sim 0.5 \mathrm{~m}$ off the bottom for a minimum of $10 \mathrm{~min}$

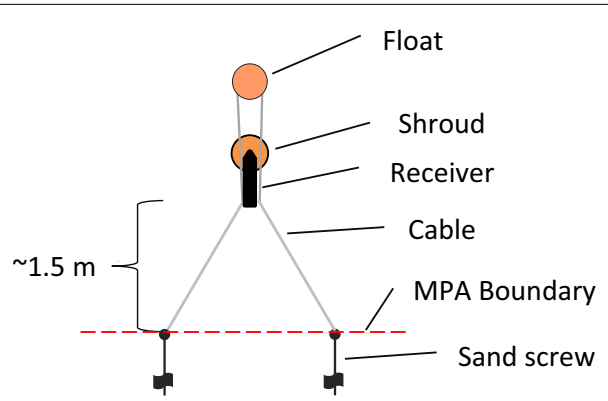

Fig. 2 Diagram of the directional mooring system to maintain receiver orientation along the Monument boundary at each range-test site. This distance off the bottom simulated the near-benthic position typical of the reef fish in the area. This transmitter was deployed repeatedly in various directions, distances, and landscape settings relative to each receiver (Fig. 3).

Distance to range-test site and detection rate (the percentage of transmissions actually detected out of the transmissions emitted at each range-test site) were plotted for those range-test sites on the shrouded versus unshrouded sides of the receiver for comparison. A detection rate of $50 \%$ was recently suggested by the manufacturer for identifying a reliable tag detection range [15] although researchers typically customize acceptable cut-offs [16]. Detection range is defined here as the shape of the curve relating detection rate and distance between receiver and tag [16]. Maximum effective detection range is defined as the distance where detection rate fell below $20 \%$. It was not possible, nor was it necessary to generate individual range-test curves for each receiver since all distances were not tested at all sites and these receivers were deployed in relatively consistent environmental settings.

A compass plot was used to evaluate detection performance relative to the shrouded versus unshrouded hemispheres of the receiver. For this, both the distance and angle of the range-test transmitter relative to the shroud was depicted. Angles were standardized in the compass plot such that the shroud theoretically blocked detections from the south. Note that the actual angles of shroud deployment at each receiver varied to be aligned with Monument boundaries (Fig. 3).

\section{Statistical analysis}

Probability of detection was modeled with a quasibinomial generalized linear model with a logit link function and where the response data were the numbers of detected and undetected pings from each rangetest site. This model offers an advantage over a simpler unweighted logistic regression in that it naturally accounts for any differences in deployment time at each range-test site. Samples of longer duration have more influence on model fit. Independent variables were distance (continuous) and shrouded versus unshrouded side of the receiver (factor). The fitted model was used to predict the probability of detection within a circle whose radius equaled the maximum distance in the data. Statistical analyses were performed in R ( R Development Core Team, http://www.R-project.org).

\section{Results}

All test sites (6/6) within $\sim 100 \mathrm{~m}$ of the receiver on the unshrouded hemisphere experienced good or excellent detection (75-100\% of possible detections) and 9 out 


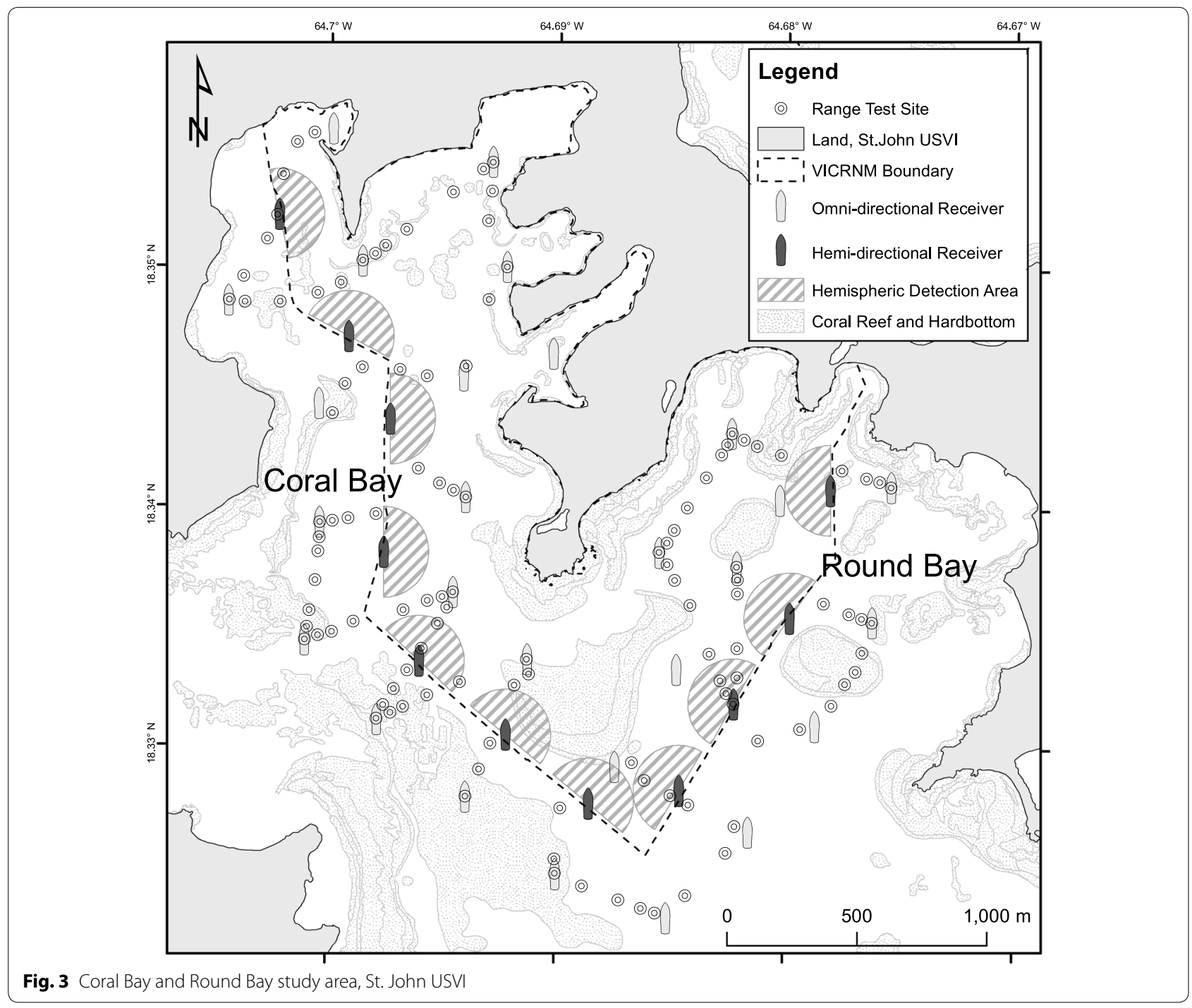

of 13 test sites within $\sim 200$ m of the unshrouded direction had at least a few transmissions detected (Fig. 4). In rare cases, detection strength was good beyond $\sim 200$ $\mathrm{m}$ away in this direction. Detection rate dropped below $50 \%$ at $\sim 125 \mathrm{~m}$, fell below $20 \%$ at $\sim 180 \mathrm{~m}$, and reached zero at all sites beyond $\sim 230 \mathrm{~m}$. These patterns were consistent with the 150-300 m detection ranges observed for omni-directional receivers that were deployed elsewhere in our study region based on similar sized [pers. obs.] or slightly larger transmitters $[17,18]$. The shape of this pattern matches the typical sigmoid or logistic shape seen in similar assessments $[6,12]$.

In contrast, detection rates were much lower in the hemisphere on the shrouded side of the receiver (Fig. 4). Dramatically fewer transmissions were detected in the direction blocked by the shroud such that the manufacturer-recommended detection rate of $50 \%$ was never achieved and only 4 out of 17 test sites within $\sim 200$ m detected any transmissions in that direction. Transmitters within $\sim 60 \mathrm{~m}$ of the receiver were detectable but never at more than a $12 \%$ detection rate and detections beyond this distance were rare.

The plot of distance, detection rate, as well as angle relative to the acoustic shroud revealed the sensitivity of shrouded receivers for detecting position near the Monument boundary (Fig. 5a). The shroud appeared effective at blocking signals along the hemispherical axis of the receivers (standardized along $90^{\circ}$ and $270^{\circ}$ in Fig. 5a, b). Transmissions emitted just $15^{\circ}$ north of this axis were readily detectable, whereas those $15^{\circ}$ south of it but at the same distance away from the receiver were generally not detectable. 


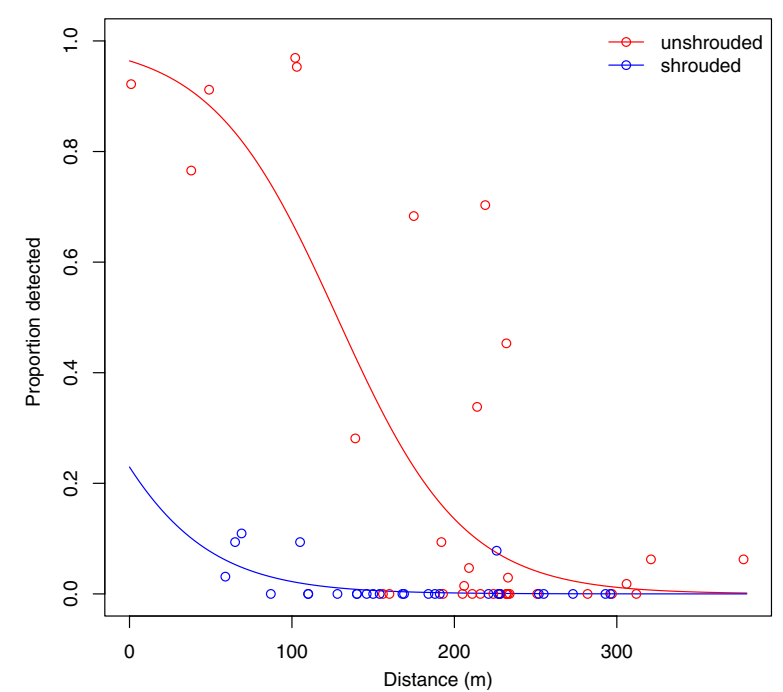

Fig. 4 Points represent the proportion of possible transmissions detected as a function of distance from the receiver to the unshrouded $(n=34)$ versus shrouded $(n=28)$ hemispheres of hemi-directional receivers. Curves denote the modeled probability of detection

The modeled probability of detection decreased with distance from the receiver $(\mathrm{p}<0.0001)$, and the shrouded and unshrouded hemispheres of the receiver exhibited significantly different probabilities of detection ( $p<0.001$; Figs. 4 and 5b). The unshrouded side of the receiver showed a rapid drop in the modeled probability of detection from 80 to $20 \%$ between $\sim 75$ and $\sim 180 \mathrm{~m}$. Based on model results, the $50 \%$ detection rate occurred at $128 \mathrm{~m}$ and a hemispherical maximum detection range of $\sim 180 \mathrm{~m}$ (corresponding to $20 \%$ probability of detection) was estimated in the unshrouded direction along the axis of the Monument boundary (Fig. 3).

\section{Discussion}

Tests here suggest that shrouded receivers can be an effective tool for improved edge detection in acoustic telemetry studies. Only transmitters on the unshrouded side of the receiver could be detected reliably at a $>50 \%$ detection rate [recommended by VEMCO, 15] or even a much more generous detection rate of $15 \%$. It is recognized that interpretation of detections and selection of appropriate cut-off values will depend on the particular setting, research questions, and behavior of the organism of interest and may differ from the $20 \%$ threshold estimated here $[1,16]$. This will require a customized set of decision rules to be established to determine the probability that an organism is on one side of a boundary or the other (e.g., [1]).

The direction of shroud placement, listening into versus out of an MPA, and other receiver placements will

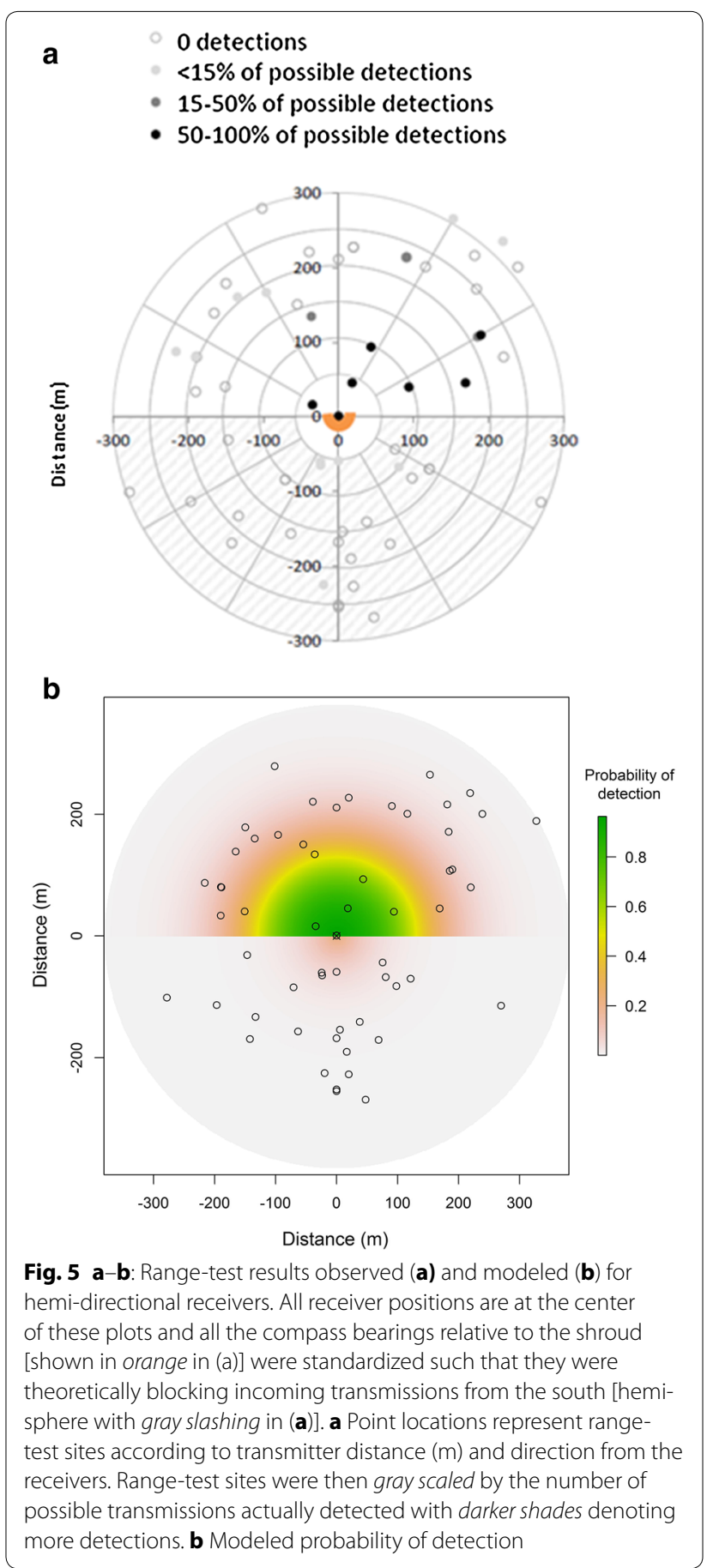

depend on the objectives of a particular study. It may be better to more conclusively detect when fish leave an MPA and are exposed to fishing than knowing that they remain safe in a given protected area. Additional omni-directional receivers could be deployed inside and outside the MPA boundary to provide a more complete understanding of directional movements (Fig. 3). This would also help with interpretation of low detection 
rates that could either be due to fish being present close by the shrouded side of the hemi-directional receiver or far away but on the unshrouded side. Although it doubles the number of receivers needed, for some applications it may also be useful to place two hemi-directional receivers on a single mooring but facing in opposite directions to determine on which side of a boundary line that an organism is located. The approach is not only useful for MPA boundary evaluation. For example, shrouded receivers can be set along linear habitat boundaries such as hardbottom/softbottom or reef/seagrass interfaces to more conclusively detect which habitat a tagged fish is utilizing. The approach may also be useful in other constrained settings where it is not desirable to detect fish presence throughout the entire circumference of an omni-directional receivers' detection range. More detailed monitoring of arrival or departure from small landscape features such as artificial reefs, spawning sites, or boat channels may also be enhanced with this technique.

It is important that shrouds be consistently shaped and snug against the transducer. Any deviations in shroud geometry should be well under the wavelength of the transmitters $(69 \mathrm{kHz}$ in this case, or $\sim 21.7 \mathrm{~cm}$ ). This will avoid irregularities in constructive or destructive interference with incoming signals on the unshrouded side of the receiver (VEMCO pers. com.). Depending on the material used to construct the shroud, an acoustically absorbent coating or randomized scattering texture may also be useful to minimize any irregular lobes or nulls in directional sensitivity.

Of course the shroud is not suitable for all applications. The approach doesn't yield position estimates, it merely provides hemispheric presence/absence for a better estimate of which side of a receiver an organism may be positioned. For detailed location and habitat utilization information, other systems are required [7-9]. Those existing approaches enable fine-scale tracking of fish position to within a few meters by deploying many receivers in high density with overlapping detection range. However, this reduces spatial coverage of a study since so many receivers must be placed in a confined area, yields much extraneous information (i.e., constant position) for applications where only boundary-crossing data is of interest, and can be cost prohibitive due to computational requirements and the large number and density of receivers that are needed.

Our analyses represent a composite value of performance for 11 receivers in a real landscape. The natural variability in landscape features present in the study area contributed to the variance in our results (Fig. 3). Two range-test sites in the unshrouded NW quadrant of Fig. 5a were within the detection range of receivers
(160 and $156 \mathrm{~m}$, respectively) but did not record any transmissions. This side of the shroud was of course identical to the other side that did have detections at similar angles and distances. We therefore looked beyond the shroud, at habitats surrounding each test location for an explanation. One of those test sites lacking detections was the shallowest and most complex reef setting in our study and the other had patch reefs nearby but unfortunately no detailed habitat information is available between the receiver and range-test site to further diagnose potential landscape interference at the second site. Therefore, although we displayed maximum detection range as a single, composite value of $\sim 180 \mathrm{~m}$ due to the relatively homogeneous environment in which most receivers were deployed, it should be recognized that detection range on the unshrouded side of individual receivers will certainly vary depending on their particular setting in the landscape $[4,13$, $15,19]$.

Our experiments were encouraging, that the shroud blocks most signal detection from one hemisphere, but do not replace the need to conduct robust range tests at each receiver site $[15,16]$. In addition, use of sentinel tags is also advisable to evaluate the long-term influence of variations in environmental noise on detection rate in most settings. Further tests are also needed to: (1) evaluate shroud performance on tagged fish now that controlled field tests have yielded positive results, (2) test the approach on data loggers available from other manufacturers, and (3) refine and evaluate other shroud materials, coatings, and designs.

\section{Authors' contributions}

MK designed and built the receiver shrouds, analyzed the data, and drafted the manuscript. MM participated in the design and execution of the study and helped to draft the manuscript. AW performed statistical analysis. All authors read and approved the final manuscript.

\section{Author details}

${ }^{1}$ NOAA/NOS/NCCOS/CCMA/Biogeography Branch, 1305 East West Highway, Silver Spring, MD 20910, USA. ${ }^{2}$ CSS-Dynamac Inc., 10301 Democracy Lane, Suite 300, Fairfax, VA 22030, USA.

\section{Acknowledgements}

Several individuals assisted with the deployment, range testing, retrieval, and download of the telemetry equipment including L Siceloff, C Cosgrove, K Roberson, B Schwartz, A Glahn, and M Kent. We thank T Kelley and NPS for boat support. L Siceloff, J Christensen, and research and development staff at VEMCO evaluated an early version of this manuscript and provided suggestions for improvement. Two anonymous peer reviewers provided constructive suggestions which also greatly improved our manuscript. Funding for this project was from NPS and NOS/NCCOS/CCMA. Government contract labor (AW) was provided by CSS-Dynamac, Inc. under NOAA contract EA-133C-14-NC-1384.

\section{Competing interests}

The authors declare that they have no competing interests.

Received: 29 September 2015 Accepted: 21 December 2015

Published online: 11 January 2016 


\section{References}

1. Knip DM, Heupel MR, Simpfendorfer CA. Evaluating marine protected areas for the conservation of tropical coastal sharks. Biol Cons. 2012;148(1):200-9.

2. Kellner JB, Tetreault I, Gaines SD, Nesbit RM. Fishing the line near marine reserves in single and multispecies fisheries. Ecol Appl. 2007;17:1039-54.

3. Hitt S, Pittman SJ, Nemeth RS. Diel movements of fishes linked to benthic seascape structure in a Caribbean coral reef ecosystem. Mar Ecol Prog Ser. 2011:427:275-91.

4. Heupel MR, Semmens JM, Hobday AJ. Automated acoustic tracking of aquatic animals: scales, design and deployment of listening station arrays. Mar Freshwater Res. 2006;57:1-13.

5. Simpfendorfer CA, Heupel MR, Hunter RE. Estimation of short-term centres of activity from an array of omnidirectional hydrophones and its use in studying animal movements. Can J Fish Aquat Sci. 2002;59:23-32.

6. How JR, de Lestang S. Acoustic tracking: issues affecting the design, analysis and interpolation of data from movement studies. Mar Freshwater Res. 2012;63:312-24.

7. Andrews KS, Tolimieri N, Williams GD, Samhouri JF, Harvey CJ, Levin PS. Comparison of fine-scale acoustic monitoring systems using home range size of a demersal fish. Mar Biol. 2011;158:2377-87.

8. Espinoza M, Farrugia TJ, Webber DM, Smith F, Lowe CG. Testing a new acoustic telemetry technique to quantify long-term, fine-scale movements of aquatic animals. Fish Res. 2011;108:364-71.

9. Cooke SJ, Niezgoda GH, Hanson K, Suski CD, Phelan FJS, Tinline R, Philipp DP. Use of CDMA acoustic technology to document 3-D positions of fish: relevance to the design and monitoring of aquatic protected areas. Mar Tech Soc J. 2005:39:17-27.

10. Clinton WJ. Establishment of the Virgin Islands coral reef national monument. Presidential Proclamation 7399. 2001.
11. Kaplan MB, Mooney TA, Partan J, Solow AR. Coral reef species assemblages are associated with ambient soundscapes. Mar Ecol Prog Ser. 2015;533:93-107

12. Hobday AJ, Pincock D. Estimating detection probabilities for linear acoustic monitoring arrays. In: Mckenzie JR, Parsons B, Seitz AC, Kopf RK, Mesa M, Phelps Q, editors. Advances in fish tagging and marking technology. Bethesda: American Fisheries Society Symposium 76; 2012. p. 325-46.

13. Mathies NH, Ogburn MB, McFall G, Fangman S. Environmental interference factors affecting detection range in acoustic telemetry studies using fixed receiver arrays. Mar Ecol Prog Ser. 2014;495:27-38.

14. Clements S, Jepsen D, Karnowski M, Schreck CB. Optimization of an acoustic telemetry array for detecting transmitter-implanted fish. N Am J Fish Man. 2005:25:429-36.

15. VEMCO. VEMCO range test software manual. DOC-5583-02. Nova Scotia; 2015.

16. Kessel ST, Cooke SJ, Heupel MR, Hussey NE, Simpfendorfer CA, Vagle S, Fisk AT. A review of detection range testing in aquatic passive acoustic telemetry studies. Rev Fish Biol Fisheries. 2014;24:199-218.

17. Friedlander AM, Monaco ME, Clark R, Pittman SJ. Fish movement patterns in Virgin Islands national park, Virgin Islands coral reef national monument and adjacent waters. Silver Spring: NOAA Technical Memorandum NOS NCCOS; 2013. p. 172

18. Pittman SJ, Monaco ME, Friedlander AM, Legare B, Nemeth RS, Kendall MS, Poti M, Clark RD, Wedding LM, Caldow C. Fish with chips: tracking reef fish movements to evaluate size and connectivity of Caribbean marine protected areas. PlosOne. 2014;9(5):e96028 (11 pp)

19. Simpfendorfer CA, Heupel MR, Collins AB. Variation in the performance of acoustic receivers and its implication for positioning algorithms in a riverine setting. Can J Fish Aquat Sci. 2008;65:482-92.

\section{Submit your next manuscript to BioMed Central and we will help you at every step:}

- We accept pre-submission inquiries

- Our selector tool helps you to find the most relevant journal

- We provide round the clock customer support

- Convenient online submission

- Thorough peer review

- Inclusion in PubMed and all major indexing services

- Maximum visibility for your research

Submit your manuscript at www.biomedcentral.com/submit

\section{(O) BioMed Central}

